\title{
Two-Stage Registration for Real-Time Deformable Compensation Using an Electromagnetic Tracking Device
}

\author{
Hui Zhang ${ }^{1}$, Filip Banovac ${ }^{1}$, Neil Glossop ${ }^{2}$, and Kevin Cleary ${ }^{1}$ \\ ${ }^{1}$ Imaging Science \& Information System (ISIS) Center, Department of Radiology, \\ Georgetown University, Washington, DC, USA \\ \{zhang, banovac, cleary\}@isis.imac.georgetown.edu \\ ${ }^{2}$ Traxtal, Inc., Bellaire, TX, USA \\ neilatraxtal.com
}

\begin{abstract}
Electromagnetic tracking systems have the potential to track instruments inside the body because they are not limited by the line of sight constraints that characterize optical tracking systems. To integrate an electromagnetic tracking device into a surgical navigation system, accurate registration is required. We present a two-stage registration mechanism designed to be more accurate than the widely used global fiducial-based registration method. The first stage uses a hybrid Iterative Closest Point (ICP) registration method and the Simulated Annealing (SA) optimization algorithm, to increase the initial registration accuracy. The second stage exploits multiple implanted tracking needles that are used to calculate the affine transform based on the initial transform information, and thereby to compensate for the deformation in real time. Phantom and swine studies have demonstrated the utility of this technique.
\end{abstract}

\section{Introduction}

While optical tracking systems are the gold standard for image-guided surgery, recent developments in electromagnetic tracking systems have enabled the development of prototype image-guided systems that can track internal organs. Electromagnetically tracked needles have been constructed that incorporate one or more electromagnetic coils in the needle stylettes. These needles can be as small as 18 gauge, and their stylettes containing the electromagnetic sensor coils can be as small as 19 gauge. Using this technology, our research group has been developing an image-guided system for abdominal interventions such as liver procedures. Liver motion due to respiration is a major concern when trying to precisely target liver lesions. The purpose of this study was to increase the accuracy of the image-guided system using a novel twostage registration method.

Fiducial and point-based registration is the most widely used registration method for image-guided surgery. In our image-guided system, fiducials are placed on the patient's skin before the pre-operative CT or MR scan. Since the flexibility of the patient's body can cause errors in registration [1], it is essential to place markers in relatively stable locations. In addition, the motion of the liver is not well correlated 
with the motion of the skin surface or underlying anatomy. Hence, external tracking of the skin surface may not be sufficient to determine the location of targets within the liver [2].

In a liver biopsy, the needle is inserted percutaneously towards the tumor target. To improve the accuracy of biopsy using our image-guided systems, our algorithm uses multiple points on a needle for registration. According to Fizpatrick [3], formula (1) is used for calculating the target registration error:

$$
\left\langle\operatorname{TRE}^{2}(r)\right\rangle \approx \frac{\left\langle F L E^{2}\right\rangle}{N}\left(1+\frac{1}{3} \sum_{k=1}^{3} \frac{d_{k}^{2}}{f_{k}^{2}}\right)
$$

where TRE is the target registration error in the position $r$ and FLE is the fiducial localization error. $N$ indicates the number of fiducials; $d_{k}$ is the distance of the target from principal axis $k$; and $f_{k}$ is the RMS distance of the fiducials. In our algorithm, the fiducial number $\mathrm{N}$ is increased by resampling the needle. Also, $d_{k} / f_{k}$ is decreased because the principal axis completed from the needle is quite close to the real target. The simulated annealing algorithm and ICP metric are integrated to give the best transformation. This transformation is used as the initial matrix for the second stage: real-time affine transform compensation. The liver and other abdominal organs are not rigid; rather, they are somewhat deformable, so the global transform is not always appropriate due to the intrinsic and extrinsic factors, such as respiration and external forces [4]. In a previous study [5], a tracked reference needle was placed inside the liver to compensate for motion, while still treating the whole model as rigid-body. Compared with the rigid body transform, the affine transform can provide additional scale and shear behavior that can be used to simulate some basic deformation. Our method uses the affine transform, which is computed in real time to reflect the exact internal motion for deformable compensation.

\section{Methods}

Our method was first tested using a respiratory motion phantom. The phantom includes a rib cage, synthetic skin, and a foam liver model that is mounted on a one degree-of-freedom precision motion platform [6]. The Aurora electromagnetic tracking system from Northern Digital Inc. was the tracking device. Tracked 18-gauge needles (MagTrax Needles, Traxtal Technologies, Bellaire, TX) were placed through the skin and into the liver to define the puncture volume. An image-guided software platform was also developed for the experiment evaluation and clinical research. The overall system implements our own registration algorithm, and also uses ITK for some registration and segmentation, VTK for visualization, and MFC for user interface. The workflow is as follows: DICOM image loading, tracker device initialization, biopsy planning, registration and segmentation, visualization, and motion tracking. The system can be used for biopsy, guide-wire deployment, verterbroplasty, and radio-frequency ablation procedures. The experiment was completed in the interventional suite at Georgetown University Medical Center. 


\subsection{First Stage: Needle Based Registration}

In order to achieve a better registration, three needles are implanted inside the liver and near the tumor target. These three needles bound a volume into which the actual procedure needle will pass through. Multiple points along these needles are used in the registration. The procedure is as follows:

\section{Algorithm 1: Needle-Based Registration}

1) Implant three non-parallel needles into the liver;

2) Obtain the pre-operative CT scan;

3) Segment the needles to obtain the image space points;

4) Sample the sensor coils embedded in the stylettes to obtain the corresponding points in tracker space;

5) Process the sampled points to reduce the noise generated by the tracking system;

6) Use ICP as the transformation metric, and integrate the simulated annealing optimization algorithm to get the correct transform.

Usually, the ICP algorithm can efficiently minimize the root mean square (RMS) distance between two feature point sets. However, it may find local minima, especially in noisy feature sets. The ICP algorithm can always reach the local minima, and the simulated annealing algorithm can achieve the global minima position slowly [7, 8]. In our algorithm, we use the ICP algorithm and a Levenberg-Marquardt solver to attain each local minima position. At these local points, the simulated annealing algorithm is applied to the transformation to perturb out and find the global one. The energy function is defined as formula (2), which is the distance between two point sets:

$$
E\left(p_{1}, \cdots, p_{M}\right)=\sum_{i=1}^{N}\left\|R A_{i}+T-C P\left(R A_{i}+T, B\right)\right\|^{2}
$$

$A$ and $B$ are the source and destination point sets. $R$ and $T$ are the rotation and translation matrices from point set $\mathrm{A}$ to $\mathrm{B}$, respectively. $C P$ is the operator that finds the closest point in another data set.

Luck and Penney have described methods that are similar to ours, but the algorithms were implemented differently $[8,9]$. Luck used the simulated annealing algorithm to produce "good" starting points for the ICP algorithm. Penny added random Gaussian noise to perturb the positions of the feature set and increase the robustness. Our method applies the simulated annealing algorithm directly to the transformation parameters of each local minimum, as described in formula (3):

$$
\left[P_{1}, \cdots, P_{M}\right]=\min \left(\operatorname{LM}\left(E\left(\tilde{p}_{1}, \cdots, \tilde{p}_{M}\right)\right)\right)
$$

$\tilde{p}_{i}$ represents the disturbed parameters around the last Levenberg-Marquardt solution position, and these parameters are used for optimization. $L M$ is the general Levenberg-Marquardt solver.

\subsection{Second Stage: Real-Time Affine Transform Compensation}

The affine transform aims to simulate the deformation in some sense, and has translation, rotation, scale, and shear operations. The anisotropic scale and shear behavior can 
be used to simulate the deformable model to some extent [10-12]. A 3D rigid-body transform has three translation factors and three rotation factors. In the affine transform, additional scale factors and other shear factors are incorporated to construct the whole matrix. The computation of the affine transform is described by Horn [13].

The three previously implanted needles are then used for compensation. When the external surgical tool is pushed into the liver body, the pre-calculated global transform will change due to the deformation behavior. Therefore, compensation is required to accurately track the target point. The standard clinical technique uses some external fiducials and internal fiducials, and tracks their positions over several respiratory cycles, trying to find the correlation between the external and internal motion [14]. Clinically, it will use only the external motion to predict the internal movement through the model. The advantage of this method is that it is non-invasive and can be effectively used to synchronize the treatment planning system. However, this method also has several drawbacks. First, it predicts the internal motion from the external motion, whose accuracy greatly depends on the predicting model, without a fixed equation or formula to validate. Second, it treats the whole body as rigid, and then applies the rigid-body compensation to the whole body's motion. Third, the method cannot compensate for the intrusion deformation caused by the surgical tools during the intervention.

The 3D affine transform has 12 defining parameters, and we only use three implanted needles for real-time tracking. Consequently, the orientation information of each needle is used to get three additional points besides three sensor points. Therefore, we have 6 points from three needles for the affine transform registration. These six points are typically chosen as the sensor points and their fixed offset points along the needle direction. The procedure is as follows:

\section{Algorithm 2: Real-Time Affine Transform Compensation}

1) At end-expiration period, record the tracking information and generate 6 points in electromagnetic space;

2) Apply the inverse of the transformation calculated in algorithm 1 to get the respective 6 points in the image space. These points are the fixed image points during the tracking;

3) Throughout the whole procedure, record the tracking needles and generate 6 different points in real-time that correspond to the first 6 points due to the deformation;

4) Use point-based registration between these changing tracking points and the fixed image points to update the affine transform in real time;

5) Apply the updated transform to the system, and repeat from step 3.

\section{Results}

The whole procedure was separated into the two stages explained above. The initial transform calculated from stage one was used for the fixed point set calculation in the second stage. We tested our algorithm with the liver phantom model. In stage one, the final transform matrix and fiducial registration error (FRE) were recorded. Since the target registration error (TRE) is more important for clinical applications, the center point of the triangle defined by the tips of the three needles is considered as the target 
point. The positions in the image space and in the electromagnetic space were recorded and compared as the TRE.

The intermediate results of the optimization procedure are listed in Table 1 and shown in Fig 1. The final optimization registration result indicates that the FRE is $0.96 \mathrm{~mm}$ and the TRE is $2.67 \mathrm{~mm}$ using our registration method. We also computed the registration matrix through the four skin-fiducials in the same case. The result of the skin-fiducial based registration indicates an FRE of $1.49 \mathrm{~mm}$ and a TRE of $7.54 \mathrm{~mm}$. Therefore, the hybrid registration method proposed here is more accurate than the skin fiducial-based registration method.

Fig. 2 shows the point sets before and after the registration method, optimized by ICP and SA algorithms. Fig. 3 shows the needle position validation result after we applied the different matrix to our system. In the left image, we can see the displacement error of the overlaid needle tip compared with the background pre-operative CT image slice. In the right image, the two tips fit together quite well using our registration algorithm.

Table 1. Process for hybrid registration of ICP and SA algorithm

\begin{tabular}{llllll}
\hline Iteration & FRE $(\mathrm{mm})$ & TRE $(\mathrm{mm})$ & Iteration & FRE $(\mathrm{mm})$ & TRE $(\mathrm{mm})$ \\
\hline 1 & 60.59 & 76.42 & 2 & 25.67 & 79.52 \\
3 & 24.82 & 80.38 & 4 & 24.36 & 80.34 \\
5 & 23.91 & 80.39 & 6 & 21.71 & 5.18 \\
7 & 15.36 & 10.75 & 8 & 3.20 & 3.46 \\
9 & 2.97 & 3.30 & 10 & 2.21 & 3.20 \\
11 & 0.96 & 2.67 & & & \\
\hline
\end{tabular}

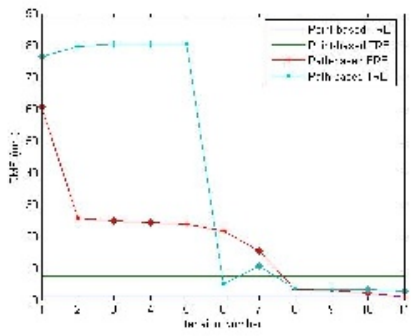

Fig. 1. Optimization process of the hybrid registration. The final registration specification of FRE and TRE is better than the skin fiducial-based registration.

To validate real-time updating of the affine transform, we implanted four needles into the phantom model. Three needles were used in the matrix computation and the fourth needle was used as the target for verification. TRE is calculated by comparing the real position reported by the tracking system and the position generated by the registration transform. Some random deformations of the liver torso by the external force and intrusion were simulated. We also provided the global registration result without affine compensation from the same first-stage transform matrix for 

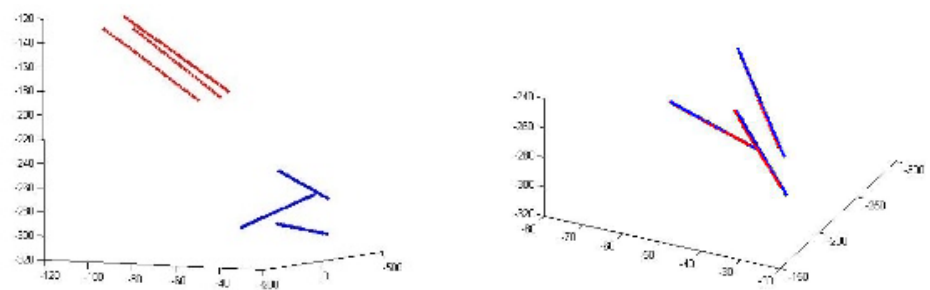

Fig. 2. Point sets before and after registration. Left: The initial position of needles in image space and position sensor space. Right: After the registration. The two point sets agree well and appear as overlaid lines.
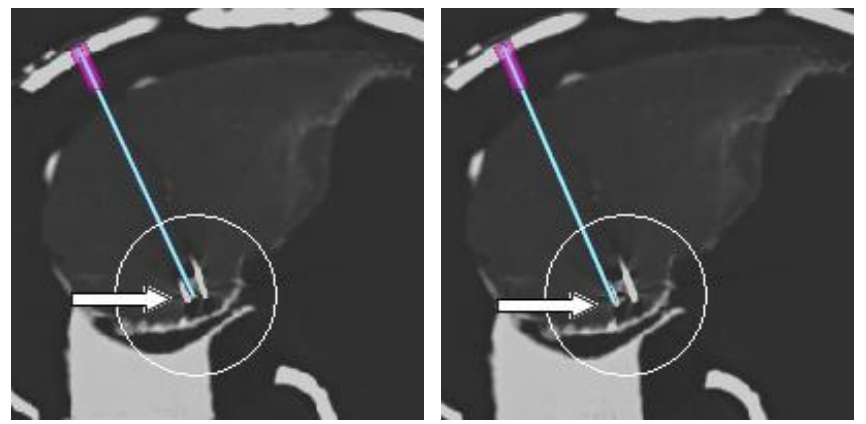

Fig. 3. Needle verification of skin fiducial-based registration and our hybrid registration method. Left: The tip of the needle is a bit off the pre-operative CT image due to the distance between the skin fiducials and target point. Right: The tip is perfectly overlaid with the left band, showing that our needle-based registration algorithm can improve the target registration accuracy.

Table 2. Comparison of the affine compensation with the global registration

\begin{tabular}{llll}
\hline & Affine TRE $(\mathrm{mm})$ & Global TRE $(\mathrm{mm})$ & Improvement $(\%)$ \\
\hline Phantom1 & $2.47 \pm 0.03$ & $2.97 \pm 0.52$ & 16.74 \\
Phantom2 & $2.18 \pm 0.44$ & $3.40 \pm 2.03$ & 36.03 \\
Swine1 & $3.30 \pm 1.16$ & $3.70 \pm 1.61$ & 10.84 \\
Swine2 & $2.07 \pm 0.70$ & $2.80 \pm 1.45$ & 26.21 \\
Swine3 & $2.14 \pm 0.87$ & $2.82 \pm 1.54$ & 24.07 \\
\hline
\end{tabular}

comparison. The results of the phantom simulation and the swine study are given in Table 2, which shows a $10.84 \%$ to $36.03 \%$ improvement in accuracy, with a standard derivation better than the global one.

A swine study was also completed under an approved protocol, with three needles for real-time affine compensation and the fourth needle for validation. Three $30 \mathrm{sec}-$ ond periods, each containing several respiratory cycles, were recorded by the tracking system. The results are shown in Fig. 4. After the registration, the liver target was successfully punctured, guided by our software as shown in Fig. 5. 

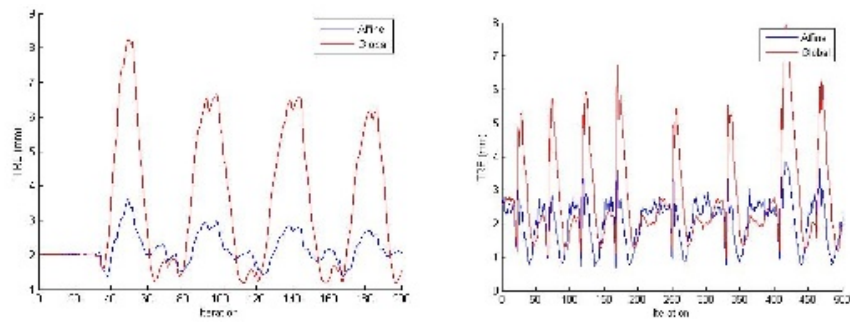

Fig. 4. TRE of affine compensation and global registration. Left: Phantom study with organ deformation. Right: Swine study with respiration deformation.

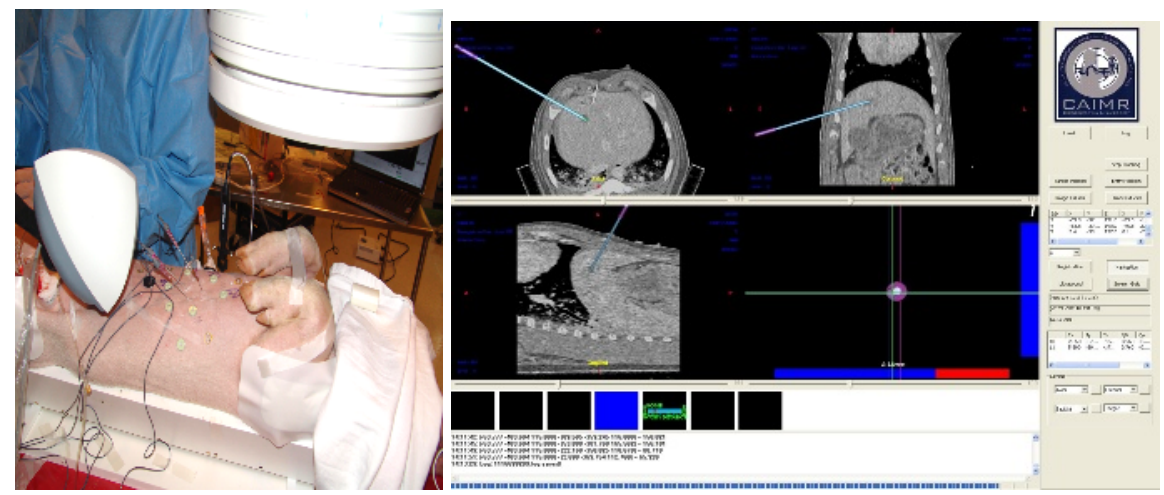

Fig. 5. Left: Image-guided tracking system in swine study. Right: graphical user interface.

\section{Discussion}

These novel registration methods may be helpful in practical applications. First, we created a hybrid registration method based on the ICP and SA algorithm, which can improve the accuracy of needle insertion. Second, we applied the position information from the tracking needles to update the real-time affine transform and compensate for the basic deformation. Compared with biomechanical model deformation, this method is simple and can be quickly completed. Finally, we proposed a two-stage strategy to combine the different registration methods together. Although we used our registration method in the first stage, any other method can be used in the first stage as the initial transform for the following calculation.

Our needle-based registration method takes advantage of position sensor readings from three reference needles and can provide better accuracy than registration based on skin fiducials. The drawback of this method is that it will take more time to calculate the initial correct transform compared to the real-time computation of point-based registration. However, once the initial transformation matrix is obtained, the affine transform can be updated in real-time to compensate for the deformation. 
Acknowledgements. This work was funded by U.S. Army grants DAMD17-99-19022 and W81XWH-04-1-0078. The content of this manuscript does not necessarily reflect the position or policy of the U.S. Government.

\section{References}

1. Rohlfing T., Maurer, C.R. Jr., O'Dell W.G., and Zhong, J., Modeling Liver Motion and Deformation during the Respiratory Cycle using Intensity-based Nonrigid Registration of Gated MR Images, Medical Physics, 31:427-432, 2004.

2. Achim Schweikard, Greg Glosser, Mohan Bodduluri, Martin Murphy, John R. Adler, Robotic Motion Compensation for Respiratory Movement during Radiosurgery, Computer Aided Surgery, 5(4):263-277, 2000.

3. Fitzpatrick J.M., West J.B., Maurer C.R., Predicting Error in Rigid-Body Point-Based Registration, IEEE Transactions on Medical Imaging, 17(5):694-702, 1998.

4. Julien Jomier, Stephen R. Aylward, Rigid and Deformable Vasculature-to-Image Registration: a Hierarchical Approach, MICCAI 2004, St Malo, France, 2004.

5. Filip Banovac, Neil D. Glossop, David Lindisch, Daigo Tanaka, Elliot Levy, Kevin Cleary: Liver Tumor Biopsy in a Respiring Phantom with the Assistance of a Novel Electromagnetic Navigation Device. MICCAI 2002, 1:200-207, 2002.

6. Kevin Cleary, Filip Banovac, Elliot Levy, Daigo Tanaka, Development of a Liver Respiratory Motion Simulator to Investigate Magnetic Tracking for Abdominal Interventions SPIE Medical Imaging 2002, 4681:25-29, 2002.

7. Zhengyou Zhang. Iterative Point Matching for Registration of Free-Form Curves and Surfaces. International Journal of Computer Vision, 13(2):119-152, 1994.

8. G. P. Penny, P.J. Edwards, A.P.King, J.M. Blackall, P.G.Batchelor, D.J.Hawkes, A Stochastic Iterative Closest Point Algorithm (stochastICP), Medical Image Computing and Computer-Assisted Intervention, MICCAI 2001, 2208: 762-769, 2001.

9. Jason Luck, Charles Little, Willam Hoff, Registration of Range Data Using a Hybrid Simulated Annealing and Iterative Closest Point Algorithm, Proc. of IEEE Int. Conf. on Rob. and Auto., San Francisco, April 24-28, 3739-3744, 2000.

10. GJ Klein, BW Reutter, RH Huesman, 4D Affine Registration Models for RespiratoryGated PET, IEEE Nuclear Science Symposium, 15:41-45, 2000.

11. J. Feldmar, N. Ayache. Rigid, affine and locally affine registration of free-form surfaces. International Journal of Computer Vision, 18:99-119, 1996.

12. Alain, Pitiot, Gregoire Malandain, Eric Bardinet, Paul M Thomposon, Piecewise Affine Registration of Biological Images, WBIR 2003, 91-101, 2003.

13. Berthold K. P. Horn, Closed-form solution of absolute orientation using unit quaternions, Journal of the Optical Society of America A, 4:629-642, 1987.

14. Ritchie CJ, Hsieh J, Gard MF, Godwin JD, Kim Y, Crawford CR., Predictive Respiratory Gating: a new Method to Reduce Motion Artifacts on CT Scans. Radiology, 190:847-852, 1994. 